\title{
Capillary refill time: the missing link between macrocirculation and microcirculation in septic shock?
}

\author{
Glenn Hernández ${ }^{1}$, Ricardo Castro ${ }^{1}$, Jan Bakker ${ }^{1,2,3,4}$ \\ ${ }^{1}$ Departmento de Medicina Intensiva, Facultad de Medicina, Pontificia Universidad Católica de Chile, Santiago, Chile; ${ }^{2}$ Department of Pulmonology \\ and Critical Care, Columbia University Medical Center, New York, NY, USA; ${ }^{3}$ Department of Pulmonology and Critical Care, NYU Medical \\ Center, Bellevue Hospital, New York, NY, USA; ${ }^{4}$ Erasmus MC University Medical Center, Rotterdam, The Netherlands \\ Correspondence to: Glenn Hernández, MD, PhD. Departmento de Medicina Intensiva, Facultad de Medicina, Pontificia Universidad Católica de \\ Chile, Santiago, Chile. Email: glennguru@gmail.com. \\ Provenance and Peer Review: This is an invited article commissioned and reviewed by the Section Editor Xue-Zhong Xing [National Cancer Center \\ (NCC)/Cancer Hospital, Chinese Academy of Medical Sciences (CAMS) and Peking Union Medical College (PUMC), Beijing, China]. \\ Response to: Yoon JC, Kim WY. Basics of changes in hemodynamic monitoring in sepsis care. J Thorac Dis 2019;11:E168-70.
}

Submitted Dec 16, 2019. Accepted for publication Jan 04, 2020.

doi: $10.21037 /$ jtd.2019.12.102

View this article at: http://dx.doi.org/10.21037/jtd.2019.12.102

\section{Introduction}

ANDROMEDA-SHOCK was a multicenter, randomized controlled trial comparing capillary refill time (CRT)versus lactate-targeted resuscitation in patients with early septic shock (1). The protocol mandated sequential steps starting with fluid challenges, followed by vasoactive-related interventions if necessary, until the target was reached. CRT-targeted resuscitation was associated with lower mortality (34.9\% vs. 43.4\%; $\mathrm{P}=0.06)$, beneficial effects on organ dysfunction, and less treatment intensity. A strong survival benefit was also supported by a recently published post-hoc Bayesian analysis (2).

The general outline of this study and its results were discussed by Yoon and Kim in a recent editorial commentary. Our purpose is to address some additional background ideas and perspectives on ANDROMEDASHOCK's results.

\section{Theoretical background}

The prognostic value of persistent hyperlactatemia in septic shock is widely recognized. However, the use of lactate as a potential resuscitation target is controversial since it is a non-specific marker of hypoperfusion that also exhibits slow recovery kinetics $(3,4)$. In fact, decreases in lactate levels even after successful resuscitation may exhibit a biphasic curve. First, a rapid initial decrease in parallel to other flow-sensitive parameters. Second, a later slower decline, probably representing residual stress-related hyperlactatemia and metabolic clearance problems $(4,5)$. Therefore, pursuing lactate normalization may lead to overresuscitation and fluid overload, which has been associated with higher mortality and worsening organ dysfunction.

On the other hand, peripheral perfusion appears to be a flow-sensitive variable that may be used to guide septic shock resuscitation (1). The skin territory lacks auto-regulatory flow control, and therefore, sympathetic activation impairs skin perfusion during circulatory dysfunction. Restoring systemic blood flow or perfusion pressure during the resuscitation process may also deactivate adrenergic response, resulting in an improvement in skin blood flow that can be captured by the CRT maneuver. This process is essentially a visible ischemia-reperfusion phenomenon that can be followed in real-time without any technologic device.

In previous observational studies, patients who didn't normalize peripheral perfusion after initial or advanced intensive care unit (ICU)-based resuscitation exhibited a $20-25 \%$ higher mortality than early normalizers (5-7). This striking difference deserves further research since it appears as counterintuitive that reperfusion of the skin, a non-vital organ in the context of shock, may have this 

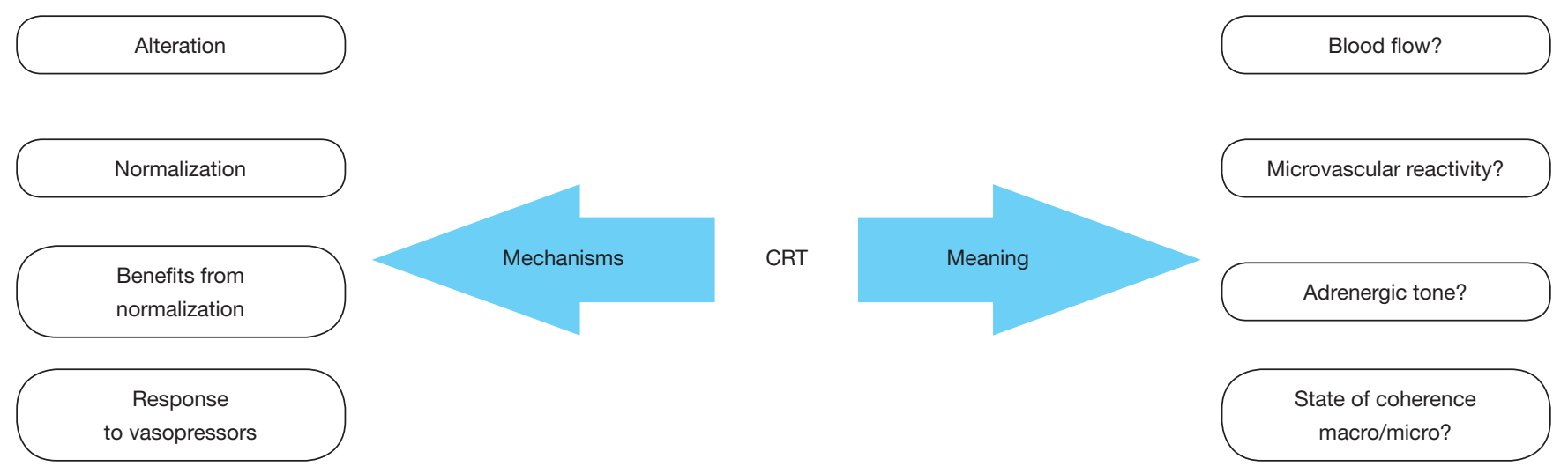

Figure 1 Scheme depicting some of the unsolved issues and challenges for a CRT-focused research agenda. Left, some of the topics to be further investigated in the mechanistic part. Right, some of the still unanswered questions regarding what CRT represents. CRT, capillary refill time.

prognostic impact. A potential explanation for the better prognosis associated with rapid CRT normalization is that it simply reflects a preserved hemodynamic coherence. Indeed, hemodynamic coherence is a condition in which resuscitation of systemic macrohemodynamic variables results in concurrent improvement in regional and microcirculatory blood flow. In early stages of septic shock, coupling between macro- and microcirculation is preserved since the predominant pathogenic mechanisms are loss of vascular tone and relative hypovolemia. Patients that rapidly normalize peripheral perfusion are probably cases of early sepsis-related circulatory dysfunction. In contrast, patients failing to improve CRT may have lost macro- and microcirculation coupling due to the interaction of more complex mechanisms such as endothelial/coagulation/ microcirculatory dysfunction. This may explain the higher mortality in CRT non-improvers $(8,9)$.

A complementary explanation could be that reperfusion of the skin simply reflects the deactivation of the neurohumoral response to stress with a decrease in adrenergic vasoconstrictive tone, a fact that may also improve flow to the hepatosplanchnic region, a far more physiologically relevant territory. Supporting this idea, a recent study found that CRT was correlated with the pulsatility index, a sonographic surrogate of vascular tone of hepatosplanchnic organs in early septic shock (10).

The improved prognosis associated with CRT normalization, its rapid-response time to fluid loading, its relative simplicity and its availability in resource-limited settings were key issues to launch the ANDROMEDASHOCK trial.

\section{Mechanistic considerations}

The mechanisms involved in the beneficial effects of CRTtargeted resuscitation in ANDROMEDA-SHOCK are not clear since the trial was not designed to address these issues. However, we could speculate on the influence of septic shock's definition. Indeed, sepsis-3 included the criteria of hyperlactatemia with the explicit objective to identify a cohort of patients with a homogeneous high risk of death. Nevertheless, this definition has been criticized since it does not consider a critical issue, the multiple pathogenic mechanisms involved in persistent hyperlactatemia. Since CRT status was not included in sepsis-3 definition, a logical assumption is that a significant proportion of septic shock patients in ANDROMEDA-SHOCK may have had normal CRT at baseline. Because of this, it is plausible that when assigned to CRT-targeted resuscitation they received less fluids and vasoactive interventions, and this may have contributed to the beneficial impact on major outcomes.

\section{Unsolved issues and challenges for a CRT- focused research agenda}

As a concept, then, the CRT has progressively and consistently shown to be a dynamic interface, standing between physiology and pathophysiology, between perfusion and flow, and perhaps between macro and microcirculation. There are still many aspects to address and research, mainly in the areas of its mechanisms and meaning (Figure 1) while we continue to learn to what extent its clinical relevance expands in the everyday ICU care. 


\section{Relevance}

Are we ready for using CRT and to abandon lactate as a resuscitation target? We strongly believe that both variables are complementary. Following the results of the ANDROMEDA-SHOCK, including the post-hoc Bayesian analysis, it appears legitimate to use CRT as a target for fluid resuscitation and to the fine-tuning of vasoactive agents. The strategy of reassessing CRT every 30 minutes until normalization is feasible and could be associated with less resuscitative interventions, a decisive factor to improve prognosis. Still, lactate evolution may provide valuable information on the general trend of septic shock resuscitation. A failure to decrease lactate levels should lead to a reevaluation of diagnosis, source control and hemodynamic/perfusion status.

\section{Acknowledgments}

Funding: None.

\section{Footnote}

Conflicts of Interest: The authors have no conflicts of interest to declare.

Ethical Statement: The authors are accountable for all aspects of the work in ensuring that questions related to the accuracy or integrity of any part of the work are appropriately investigated and resolved.

Open Access Statement: This is an Open Access article distributed in accordance with the Creative Commons Attribution-NonCommercial-NoDerivs 4.0 International License (CC BY-NC-ND 4.0), which permits the noncommercial replication and distribution of the article with the strict proviso that no changes or edits are made and the original work is properly cited (including links to both the formal publication through the relevant DOI and the license). See: https://creativecommons.org/licenses/by-nc-nd/4.0/.

Cite this article as: Hernández G, Castro R, Bakker J. Capillary refill time: the missing link between macrocirculation and microcirculation in septic shock? J Thorac Dis 2020;12(3):1127-1129. doi: 10.21037/jtd.2019.12.102

\section{References}

1. Hernández G, Ospina-Tascón GA, Damiani LP, et al. Effect of a resuscitation strategy targeting peripheral perfusion status vs serum lactate levels on 28-day mortality among patients with septic shock: the ANDROMEDA-SHOCK randomized clinical trial. JAMA 2019;321:654-64.

2. Zampieri FG, Damiani LP, Bakker J, et al. Effects of a resuscitation strategy targeting peripheral perfusion status vs serum lactate levels among patients with septic shock: a bayesian reanalysis of the ANDROMEDA-SHOCK trial. Am J Respir Crit Care Med 2020;201:423-9.

3. Hernandez G, Bruhn A, Castro R, et al. The holistic view on perfusion monitoring in septic shock. Curr Opin Crit Care 2012;18:280-6.

4. Hernandez G, Bellomo R, Bakker J. The ten pitfalls of lactate clearance in sepsis. Intensive Care Med 2019;45:82-5.

5. Hernandez G, Luengo C, Bruhn A, et al. When to stop septic shock resuscitation: clues from a dynamic perfusion monitoring. Ann Intensive Care 2014;4:30.

6. Lara B, Enberg L, Ortega M, et al. Capillary refill time during fluid resuscitation in patients with sepsis-related hyperlactatemia at the emergency department is related to mortality. PLoS One 2017;12:e0188548.

7. Hernandez G, Pedreros C, Veas E, et al. Evolution of peripheral vs metabolic perfusion parameters during septic shock resuscitation. A clinical-physiologic study. J Crit Care 2012;27:283-8.

8. Hernández G, Teboul JL. Is the macrocirculation really dissociated from the microcirculation in septic shock? Intensive Care Med 2016;42:1621-4.

9. Hernández G, Bakker J. Perspectives on perfusion monitoring in septic shock after the ANDROMEDASHOCK trial. Med Intensiva 2019;43:578-81.

10. Brunauer A, Koköfer A, Bataar O, et al. Changes in peripheral perfusion relate to visceral organ perfusion in early septic shock: a pilot study. J Crit Care 2016;35:105-9. 